Wathematical \& Computationai Apphications. Vol. 4. No. 3. pp. 241-249. 1999

(1). Association for Scientific Research

\title{
PLANE WAVE DIFFRACTION BY AN OPEN \\ PARALLEL PLATE WAVEGUIDE WITH DIELECTRIC LOADING
}

\author{
Bahattin Türetken $^{1} \quad$ Ali Alkumru ${ }^{2}$ \\ ${ }^{1}$ TÜBITAK -UEKAE P:O Box.41.41470 \\ Gebze, Kocaeli, TURKEY \\ ${ }^{2}$ Faculty of Engineering Gebze Institute of Technology P.O.Box. 141, 41400 \\ Gebze, Kocaeli, TURKEY
}

\begin{abstract}
The diffraction of plane electromagnetic waves by an open parallel plate waveguide with dielectric loading is investigated rigorously by using the Fourier transform technique. This method of formulation gives rise to Wiener- Hopf equations. The solution of each Wiener-Hopf equation contains a set of infinitely many constants satisfying an infinite system of linear algebraic equations. A numerical solution of this systems is obtained for various values of the distances between the plates, plate thickness, the number of wave in the dielectric region.
\end{abstract}

\section{INTRODUCTION}

The scattering of plane waves by a series of parallel plates constitutes an important class of canonical problem in diffraction theory and has been studied for many years.

The scattering by a parallel plate waveguide $[1,2]$ and by an infinite grating [3.4]. [5] was resolved in the early days but other configurations such as three parallel half-planes have long defied analysis. The reason is that the solution rests on the Wiener-Hopf technique.

The diffraction of plane waves by three parallel infinitely thin soft half-planes has been considered first by D.S. Jones who formulated the problem as a three dimensional matrix Wiener-Hopf equation [6]. The three parallel half-planes problem has been also considered by Abrahams [7] who presented a more simples approach to achieve the Wiener-Hopf factorisation of the Kernel matrix.

We consider the diffraction of Ez-polarized plane waves by an open parallel plate waveguide[8]

The traditional formulation of this problem leads to Wiener-Hopf equation which can not be solved by considering the known techniques.

A numerical solution of this system is obtained for various values of the wave number of dielectric region plate thicknesses and distance between the plates, through which the effect of these parameters on the diffraction phenomenon are studied.

A time factor $e^{-t \omega t}$ with $\omega$ being the angular frequency is assumed and suppressed throughout the paper. 


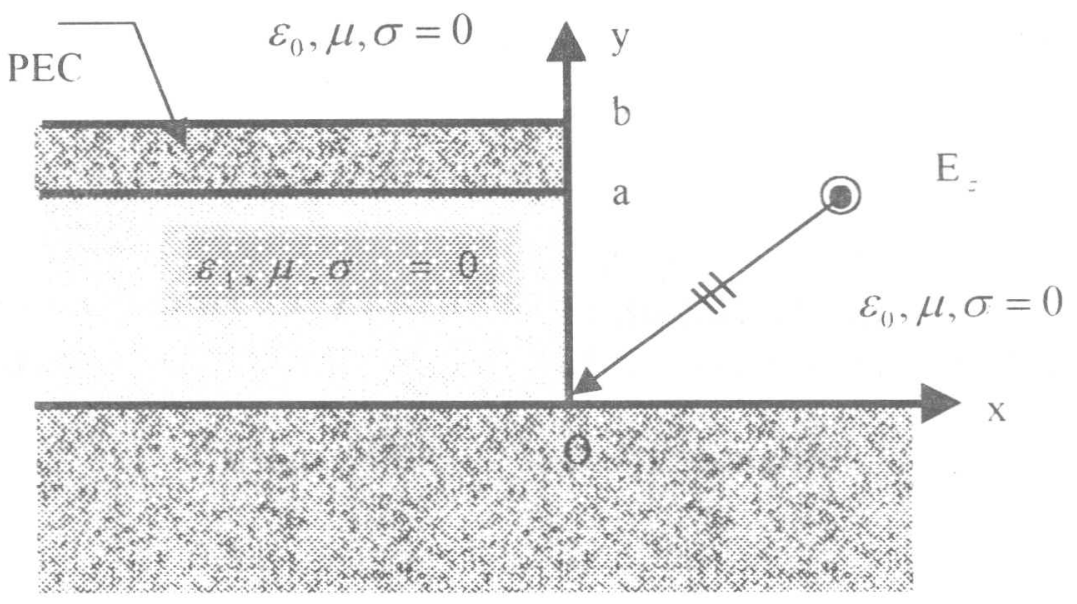

Fig.1 Geometry of the problem

\section{ANALYSIS}

We consider the diffraction of an $E_{z}$-polarized plane wave by a thick semi-infinite plate defined by $S=\{(x, y, z) ; x \in(-\infty, 0), y \in(a, b), z \in(-\infty, \infty)$ located over a perfectly conducting plane as shown in Fig. 1 .

The waveguide is supposed to be filled by a dielectric material with constitutive parameters $\varepsilon=\varepsilon_{1}, \mu=\mu_{0}$ and $\sigma=0$.

For analysis purposes, it is convenient to express the total field as follows:

$$
\mathrm{U}_{\mathrm{T}}= \begin{cases}u^{\prime}(x, y)+u^{r}(x, y)+u_{1}(x, y), & y>b \\ u_{2}(x, y) & ; 0<y<a, x<0 \\ u_{3}(x, y) & ; 0<y<b, x>0\end{cases}
$$

Here, $u^{i}$ is the incident field given by

$$
u^{i}(x, y)=\exp \left(-i k\left(x \cos \phi_{0}+y \sin \phi_{0}\right)\right)
$$

while $u^{r}$ denotes the field reflected from the plane $\mathrm{y}=\mathrm{b}$, namely

$$
u^{r}(x, y)=-\exp \left(i k\left(x \cos \phi_{0}-(y-2 b) \sin \phi_{0}\right)\right)
$$

In $(2 a, b) k$ is the free space wave number which is assumed to have a small positive imaginery part: $k_{l}$ is the wave number of dielectric region. $u_{j}, \mathrm{j}=1,2,3$, which satisfy the

$$
\begin{aligned}
& \Delta u_{1}+k^{2} u_{i}=0 \\
& \Delta u_{2}+k_{l}^{2} u_{2}=0 \\
& \Delta u_{3}+k^{2} u_{3}=0
\end{aligned}
$$

Helmholtz equation in appropriate regions are to be determined with the aid of the following boundary and continuity conditions 


\section{BOUNDARY CONDITIONS AND WIENER HOPF EQUATION}

The total field $\mathrm{U}_{\mathrm{T}}(\mathrm{x}, \mathrm{y})$ whic satisfy the Helmholtz equation is to be determined with the aid of following boundary and contiunity conditions:

$$
\begin{aligned}
& u_{l}(x, b)=0 ; x \quad 0 \\
& u_{2}(x, a)=0 ; x \quad 0 \\
& u_{2}(x, 0)=0 ; x \quad 0 \\
& u_{3}(x, 0)=0 ; x \quad 0 \\
& u_{1}(x, b)-u_{3}(x, b)=0 ; x \quad 0 \\
& \frac{\partial u_{i}}{\partial y}(x, h)-\frac{\partial u_{3}}{\partial y}(x, h)=2 i k \sin \phi_{0} e^{-i k b \sin \phi_{i \prime}} e^{-l k x \cos \phi_{i \prime}} ; x \quad 0 \\
& u_{2}(0, y)=u_{3}(0, y): 0 \quad y \quad a \\
& \frac{\partial u_{2}}{\partial x}(0, y)=\frac{\partial u_{3}}{\partial x}(0, y) ; 0 \quad y \quad a \\
& u_{3}(0, y)=0 \quad ; a \quad y \quad b
\end{aligned}
$$

Since $u_{l}(x, y)$ satisfies the Helmholtz equation in the range $x \varepsilon(-x, x)$ its Fourier transform with respect to $x$ gives

$$
\left[\frac{d^{2}}{d y^{2}}+\left(k^{2}-\alpha^{2}\right)\right] F(\alpha, y)=0
$$

with

$$
F(\alpha, y) \quad F+(\alpha, y)+F \cdot(\alpha, y)
$$

where

$$
F_{+}(\alpha, y)=\int_{0}^{\infty} u(x, y) e^{t \alpha x x} d x
$$

By taking into account the following asymtotic behaviours of $u$ for $x \rightarrow \pm \infty$

$$
u_{1}(x, y)=\left\{\begin{array}{l}
0\left(e^{-i k x}\right) ; x \rightarrow-\infty \\
0\left(e^{-i k x \cos \phi_{n}}\right) ; x \rightarrow+\infty
\end{array}\right\}
$$

One can show that $F_{+}(\alpha, y)$ and $F_{-}(\alpha, y)$ are regular fonctions of $\alpha$ in the half planes $\operatorname{Im}\{\alpha\} \operatorname{Im}\left\{k \cos \phi_{0}\right\}$ and $\operatorname{Im}\{\alpha\} \operatorname{Im}\{k\}$, respectively. The general solution of (5a) satisfying the radiation condiction for $y \rightarrow \infty$ reads

$$
F_{+}(\alpha, y)+F_{-}(\alpha, y)=A(\alpha) e^{i K(\alpha)(y-b)}
$$


with

$$
K(\alpha)=\sqrt{k^{2}-\alpha^{2}}
$$

The square-root function is defined in the complex $\alpha$-plane cut along $\alpha=\mathrm{k}$ to $\alpha=\mathrm{k}+\mathrm{i} \propto$ and $\alpha=-\mathrm{k}$ to $\alpha=-\mathrm{k}-\mathrm{i} \infty$, such that $K(0)=k$.

In the Fourier transform domain (4a) takes the form

$$
F .(\alpha, b)=0
$$

By using the derivative of (7a) with respect to y and (8), we get,

$$
A(\alpha)=F \cdot(\alpha, b)
$$

In the region $0<\mathrm{y}<\mathrm{b}$ and $\mathrm{x}>0, w_{3}(x, y)$ satisfies the Helmholtz equation

$$
\left(\frac{\partial^{2}}{\partial x^{2}}+\frac{\partial^{2}}{\partial y^{2}}+k^{2}\right) u_{3}(x, y)=0
$$

The half-range Fourier transform of (10) yields

$$
\left[\frac{d^{2}}{d y^{2}}+K^{2}(\alpha)\right]\left(J_{+}(\alpha, y)=\frac{1}{2 \pi}[f(y)-i \alpha g(y)]\right.
$$

with

$$
f(y)=\frac{\partial}{\partial x} u_{3}(0, y), g(y)=u_{3}(0, y)
$$

$G_{+}(\alpha, y)$, which is defined by

$$
G_{+}(\alpha, y)=\frac{1}{2 \pi} \int_{0}^{\infty} w_{3}(x, y) e^{i v x} d x
$$

is a function regular in the half-plane $\operatorname{Im}\{\alpha\} \operatorname{Im}\{-\boldsymbol{k}\}$. The general solution of (11a) satisfying the Dirichlet boundary condition at $y=0$ reads

$$
G_{+}(\alpha, y)=B(\alpha) \sin K(\alpha) y+\frac{1}{2 \pi K(\alpha)} \int_{0}^{y}[f(t)-i \alpha g(t)] \sin \{K(\alpha)(y-t)\} d t
$$

Combining (4e) and (4f), we get

$$
F_{+}(\alpha, b)=G_{+}(\alpha, b)
$$

and $B(\alpha)$ can be solved uniquely to give

$$
B(\alpha)=\frac{F_{+}(\alpha, b)-\frac{1}{2 \pi K(\alpha)} \int_{0}^{b}[f(t)-i \alpha g(t)] \sin \{K(\alpha)(b-t)\} d t}{\sin [K(\alpha) b]}
$$

RepFacing (15) into (13) we get 


$$
\begin{gathered}
G_{+}(\alpha, y)=\frac{\sin K(\alpha) y}{\sin [K(\alpha) b]}\left\{F_{+}(\alpha, b)-\frac{1}{2 \pi K(\alpha)} \int_{0}^{b}[f(t)-i \alpha g(t)] \sin \{K(\alpha)(b-t)\} d t\right\} \\
+\frac{1}{2 \pi K(\alpha)} \int_{0}^{y}[f(t)-i \alpha g(t)] \sin \{K(\alpha)(y-t)\} d t
\end{gathered}
$$

Although the left-hand side of (16) is regular in the upper half-plane $\operatorname{Im}\{\alpha\} \quad \operatorname{Im}\{-k\}$ the regularity of the right-hand side is violated by the presence of simple poles occuring at the zeros of $\sin \left[K_{m} b\right]$ namely at $\alpha=\alpha_{m}$ satisfying

$$
\sin \left[K_{m} b\right]=0, \operatorname{Im}\{\alpha\} \cdot \operatorname{Im}\left\{-k_{\}}, \quad m=1,2, \ldots\right.
$$

These poles can be eliminated by imposing that their residues are zero This gives

$$
F_{+}\left(\alpha_{m}, b\right)=\frac{(-1)^{m+1}}{2 \pi K_{m}} \frac{b}{2}\left[f_{m}-i \alpha_{m} g_{m}\right]
$$

where

$$
K_{m}=\sqrt{k^{2}-\alpha_{m}^{2}},\left[\begin{array}{l}
f_{m} \\
g_{m}
\end{array}\right]=\frac{2}{b} \int_{0}^{b}\left[\begin{array}{l}
f(t) \\
g(t)
\end{array}\right] \sin \left[K_{m} t\right] d t
$$

Consider now the region $0 \quad y \quad a, x \quad 0$ where tha total field can be expressed in terms of Fourier series as

$$
\because \quad u_{2}(x, y)=\sum_{n=T}^{\infty} C_{n} \sin \gamma_{n} y_{e^{-i \beta m x}}
$$

with

$$
\begin{gathered}
\gamma_{n}=\frac{n \pi}{a} \\
\beta_{n}=k_{l} \sqrt{1-\left(\frac{n \pi}{k_{l} a}\right)^{2}}
\end{gathered}
$$

From the continuity relations $(4 \mathrm{~g}, \mathrm{~h})$ and $(11 \mathrm{~b}, \mathrm{c})$ we get

$$
u_{2}(\theta, y)=g(y), \frac{\partial}{\partial x} u_{2}(0, y)=f(y), \quad 0 \quad y \cdot a
$$

Owing to (18c) can be expanded into Fourier Cosine series as follows:

$$
\therefore\left[\begin{array}{l}
f(y) \\
g(y)
\end{array}\right]=\sum_{m=r}^{\infty}\left[\begin{array}{l}
f_{m} \\
g_{m}
\end{array}\right] \sin \left[K_{m} y\right]
$$

Substituting (19a) and (21) in to $(20, b)$

We obtain

$$
\sum_{m=1}^{\infty} f_{m} \sin \frac{m \pi}{y} y=-i \sum_{n=1}^{\infty} C_{n} \beta_{n} \sin \gamma_{n} y ; 0 \quad y \quad a
$$




$$
\sum_{m=1}^{\infty} g_{m} \sin \frac{m \pi}{b} y=\left\{\begin{array}{llll}
0 & ; a & y & b \\
-i \sum_{n}^{\infty}\left({ }_{l}{ }_{n} \beta_{n} \sin \gamma_{n} y ; 0\right. & y & a
\end{array}\right.
$$

Let us multiply both sides of (22) by $\sin \frac{n \pi}{a} y$ and integrate from $y=0$ to $y=$ a to get

$$
C_{m}=\frac{2}{a}(-1)^{m+1} i \frac{\gamma_{m}}{\beta_{m}} \sum_{j=1}^{\infty} f_{j} \sin \frac{j \pi a}{b} \frac{1}{\gamma_{m}^{2}-K_{j}^{2}}
$$

Similarly, the multiplication of both sides of (23) by $\sin \frac{n \pi}{b} y$ and its integration from $\mathrm{y}=0$ to $\mathrm{y}=\mathrm{b}$ yields

$$
g_{n}=i \frac{t}{a b} \sin \frac{n \pi a}{b} \sum_{m=1}^{\infty} \frac{\gamma_{m}^{2}}{\beta_{m}\left(\gamma_{m}^{2}-K_{n}^{2}\right)}\left(\sum_{j}^{\infty} f_{j} \sin j \frac{\pi a}{b} \frac{l}{\gamma_{m}^{2}-K_{j}^{2}}\right)
$$

Consider the continuity relation (4f) which reads, in the Fourier transform domain

$$
\dot{F} .(\alpha, b)-\dot{G} .(\alpha, b)=\frac{-k \sin \phi_{0}}{\pi} e^{-i k b \sin \phi_{11}} \frac{1}{\left(\alpha-k \cos \phi_{n}\right)}
$$

where the dot (.) specifies the derivative with respect to y. Taking into account (7a), (9) and (16), one obtains

$$
\begin{aligned}
& \frac{K(\alpha) e^{i k i(\alpha) h}}{\sin [K(\alpha) b]} F(\alpha, b)+\dot{F}(\alpha, b)=\frac{k \sin \phi_{0} e^{-i k b \sin \phi_{n}}}{\pi\left(\alpha-k \cos \phi_{0}\right)}-\frac{1}{2 \pi \sin [K(\alpha) b]} \times \\
& {\left[\int_{0}^{b} f(t) \sin [K(\alpha) t] d t-i \alpha \int_{0}^{b} g(t) \sin [K(\alpha) t] d t\right]}
\end{aligned}
$$

Substituting (21) in (27) and evaluating the resultant integral, one obtains the following Wiener-Hopf Equation of the second kind valid in the strip $\operatorname{Im}\left\{k \cos \phi_{0}\right\} \operatorname{Im}\{\alpha\} \quad I_{m}\{k\}$.

$\underline{Q}(\alpha) F_{+}(\alpha, b)+\dot{F}_{-}(\alpha, b)=\frac{k \sin \phi_{0} e^{-i k b \sin \phi_{\prime \prime}}}{\pi\left(\alpha-k \cos \phi_{0}\right)}-\frac{i}{2 \pi} \sum_{m=1}^{\infty} \frac{f_{m}-i \alpha g_{m}}{\left(\alpha \cdot \alpha_{m}\right)\left(\alpha-\alpha_{m}\right)} \sin \left(K_{m} b\right)$

with

$$
Q(\alpha)=\frac{e^{-i K^{\prime}(\alpha) b} K(\alpha)}{\sin [K(\alpha) b]}, \quad \alpha_{m}=\sqrt{k^{2}-K_{m}^{2}}
$$

Here $Q_{+}(\alpha)$ and $Q_{-}(\alpha)$ are the split functions, regular and free zeros in the half-planes $\operatorname{Im}\{\alpha\} \operatorname{Im}\{-k\}$ and $\operatorname{Im}\{\alpha\} \quad \operatorname{Im}\{k\}$ respectively, resulting from the Wiener-Hopf factorization of the kernel function $\frac{1}{Q(\alpha)}$ as

$$
\frac{1}{Q(\alpha)}=\frac{1}{Q_{+}(\alpha)} \frac{1}{Q-(\alpha)}
$$

The explicit expression of $Q_{\mp}(\alpha)$ can be obtained by following the procedure outlined in [2]. 
$\underline{Q}(\alpha)=\frac{\sqrt{K(\alpha)}}{\sqrt{\sin K(\alpha) b}} \exp \left\{\frac{-K(\alpha) b}{\pi} \ln \left(\frac{\alpha+i K(\alpha)}{k}\right) \exp \left(-i \alpha \frac{b}{\pi}\left(1-\left(\cdot+\ln \frac{2 \pi}{K(\alpha)}+i \pi / 2\right)\right)\right\}\right.$

$\coprod_{n}\left(1+\frac{\alpha}{\alpha_{n}}\right) e^{n-1 \alpha h}$

$$
\underline{Q}(\alpha)=\underline{()}(-\alpha), \alpha_{n}=\frac{n \pi}{a}
$$

In (3 la) $\mathrm{C}$ is the Euler's constant given by $\mathrm{C}=0,57721 \ldots$

\section{ANALYSIS OF THE FIELD AND COMPUTATIONAL RESULTS}

The scattered field in the region $\mathrm{y}>\mathrm{b}$ can be obtained by taking the inverse Fourier transform of $F(\alpha, y)$

$$
u_{i}(x, y)=\frac{1}{2 \pi} \int_{l} A(\alpha) e^{i K i(\alpha)(y-h)} e^{-i \alpha \alpha} d \alpha
$$

Here $L$ is a straight line parallel to the real $\alpha$-axis lying in the strip $\operatorname{Im}\left(k \cos \phi_{0}\right) \operatorname{Im}(\alpha) \operatorname{Im}(k)$. The asymptotic evaluation of the integrals in (32a) through the saddle point technique enables us to write for the diffraction field

$\left.u_{l}(\rho, \phi)_{-}\left\{u_{0} I\right)\left(\phi, \phi_{0}\right)+\frac{e^{i \pi+}}{+\sqrt{2} \pi^{3 \cdot 2}} \frac{k \sin \phi}{(-(-k \cos \phi)} \sum_{m=1}^{\infty} \frac{\left.\sin / K_{m} b\right]}{\alpha_{m}\left(-\left(-\alpha_{m}\right)\right.} \frac{\left(f_{m}+i \alpha_{m} g_{m}\right)}{\left(\alpha_{m}-k \cos \phi\right)}\right\} \frac{e^{i k p}}{\sqrt{k \rho}}$

$$
\begin{aligned}
& u_{0}=e^{-i k h \sin \phi_{n}} \\
& D\left(\phi, \phi_{0}\right)=\frac{-k e^{-i \pi}+}{\sqrt{2 \pi}} \frac{\sin \phi_{0}}{\left(-\left(k \cos \phi_{0}\right)\right.} \frac{\sin \phi}{\left(\cos \phi+\cos \phi_{0}\right)} \frac{1}{Q_{+}(-k \cos \phi)}
\end{aligned}
$$

where $(\rho, \phi)$ are the cylindrical polar coodinates defined by $x=\rho \cos \phi, y-b=\rho \sin \phi$ and $u_{0}$ is the expression of the incident field at $y=b, x=0$.

The results here applied to some numerical examples, which permit us grasp the effect of various parameters on the diffraction phenomena.

Fig. 2 shows the variation of the scattering field versus the observation angle for the thick of the plate(b-a). It is seen that scattering field becomes insensitive to the observation angle bigger than 100 degree.

Fig. 3 depicts the influence of the wave number of the dielectric region on the scattering field. phenomenon.

Finally, Fig.4 illustrates the effects of the incident fields on the scattering 


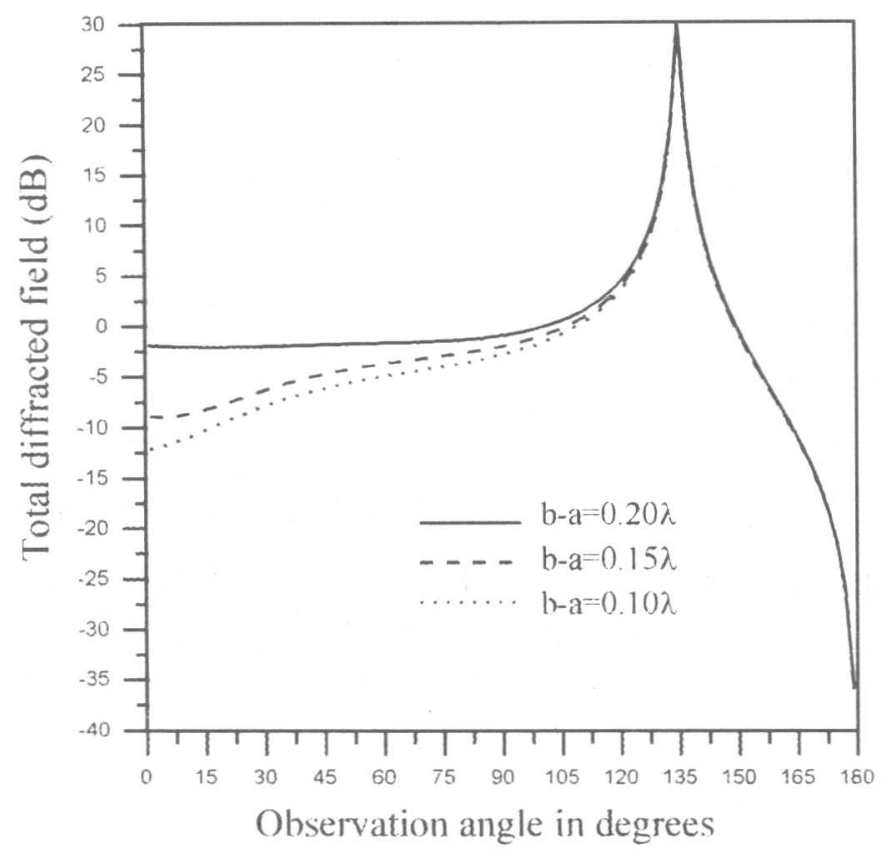

Fig 2. Scattering field versus the observation angle for different values of the plate thick (b-a)

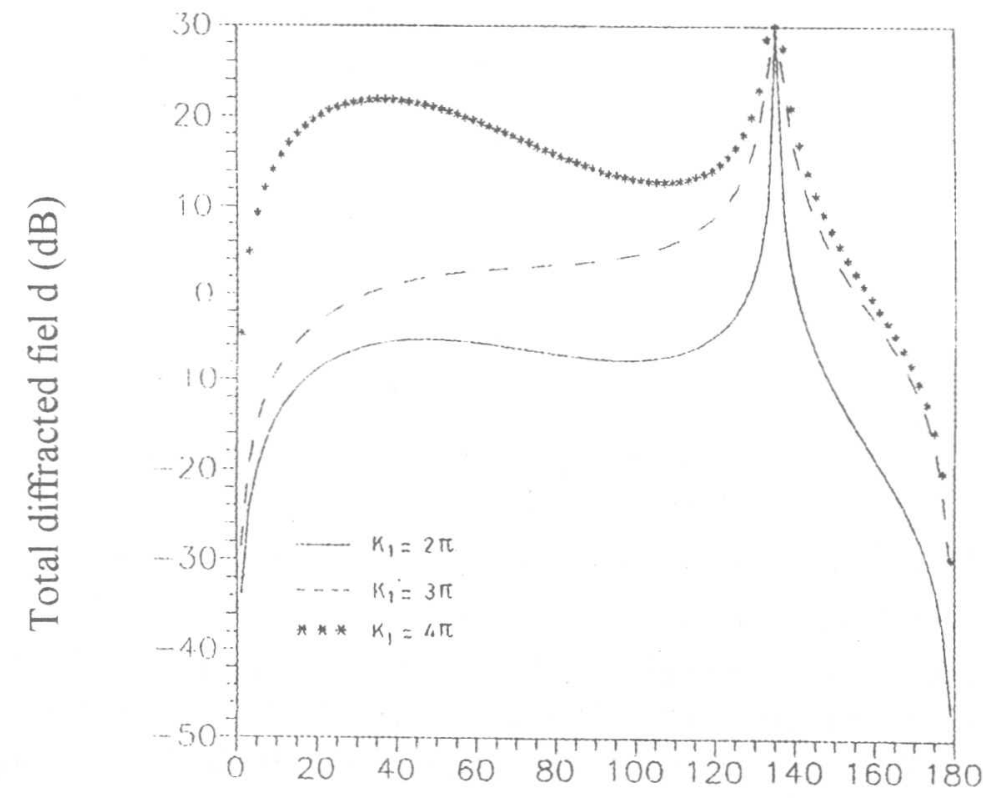

Observation angle in degrees

Fig 3. Scattering field versus the observation angle for different values of the $k_{1}$ 


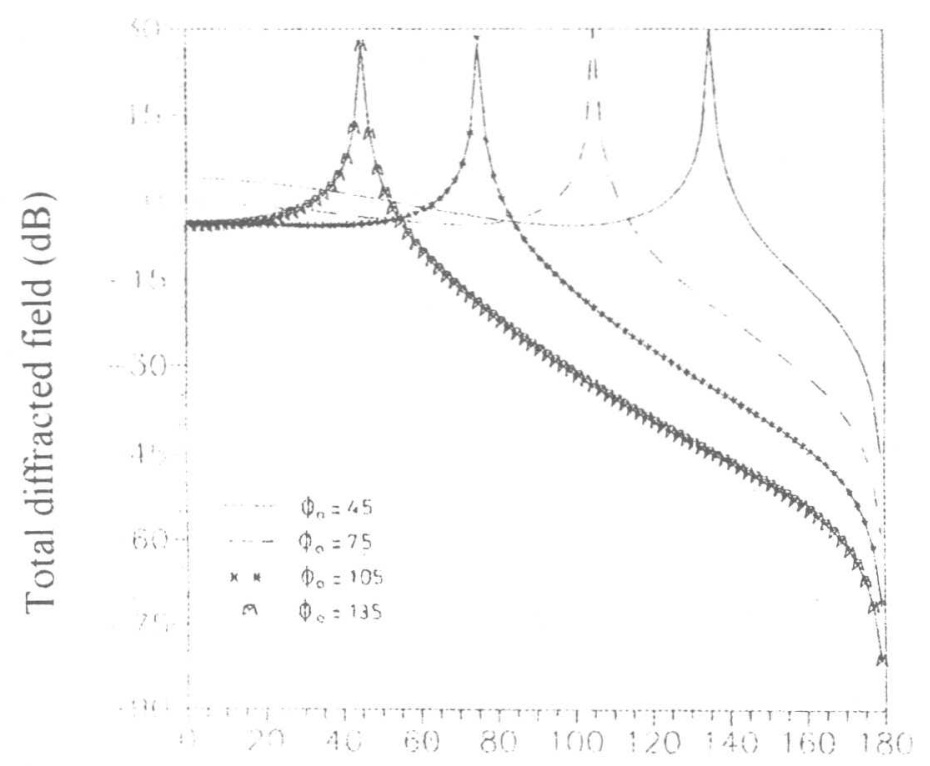

Observation angle in degrres

Fig 4. Scattering field versus the observation angle for different values of the incident fields

\section{ACKNOWLEDGMENT}

This work is partially supported by the National Research Council of Turkey-TIBITAK

\section{REFERENCES:}

1. L.A. Weinstein. The Theory of Miffratton athe Factomation Methot. Golem Press,Boluder, Colo, 1969

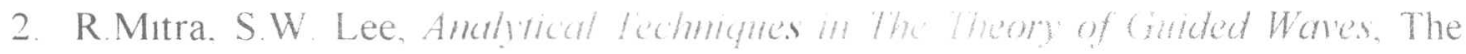
MacMillan Company, Newyork. 197 :

3. J.F. Carlson and A.E. Heins. The Reflection of an Electromagnetic Plane Wave by an Infinite Set of Plates 1, Quart.App.Math., Vol 5.82-88,1947.

4. A.E.Heins and J.F. Carlson. The reflection of an Electromagnetic Plane Wave by an Infinite Set of Plates II.Quart.Appl.Math., Vol 5,82-88, 1947.

5. A Büyükaksoy and B.Polat. Plane Wave Diffraction by a Thick Walled Parallel Plate Impedance Waveguide, HIEL; Trans.Amenims and I'ropragat. Vol.46. December 1998.ISSUE

6. B. Jones, Diffication by Three Semi-Infinite Planes, J Sound., A404,299-321, 1986

7. I.D.Abrahams, Scattering of Sound by Three Semi-Infinite Planes, J.Sound Vibr.,112,396-398, 1987.

8. B. Türetken, Plane Wave Diffraction By An Open Parallel Plate Waveguide, XXVT th (ieneral Assembly of the International Inion of Radio Sicience August 1321,1999, Toronto, Canada 